\title{
Etude de deux méthodes d'application du triflumuron sur les mâles de Glossina palpalis gambiensis au laboratoire : impact sur la reproduction des femelles
}

\author{
Z.A. Bancé ${ }^{1,4}$ P.A. Ouedraogo ${ }^{2}$ \\ B. Bauer ${ }^{3}$ I. Kaboré ${ }^{4}$ I. Sidibé ${ }^{4}$
}

Mots-clés

Glossina palpalis gambiensis Triflumuron - Régulateur croissance insecte - Application locale Pulvérisation - Burkina Faso.

\begin{abstract}
Résumé
L'objectif de cette étude a été de comparer l'efficacité de deux méthodes d'application du triflumuron sur des glossines mâles pour le transfert du produit aux femelles pendant l'accouplement. La méthode topique, avec dépôt de la solution sur la partie supérieure du thorax, a été utilisée pour quatre groupes de 150 glossines mâles âgées de six jours ayant reçu $1 \mu \mathrm{g}$, $2 \mu \mathrm{g}$ et $5 \mu \mathrm{g}$ de triflumuron contenu dans $1 \mu \mathrm{l}$ d'eau, et le témoin ayant reçu de l'eau. Chaque groupe de mâles a été ensuite successivement accouplé avec trois groupes de 150 femelles âgées de trois jours pendant $48 \mathrm{~h}$. Les effets mesurés ont été les taux d'avortement et d'éclosions des pupes, la forme et le poids des pupes. L'analyse des résultats sur les quatre premiers cycles de reproduction des femelles a montré des différences significatives entre les taux d'éclosions chez les femelles accouplées avec les mâles traités aux doses de $2 \mu \mathrm{g}$ et $5 \mu \mathrm{g}$ et les taux d'éclosions chez les femelles du lot témoin, uniquement au premier cycle du premier accouplement $(p<0,05)$. La deuxième méthode a consisté à asperger un jet de $0,8 \mathrm{ml}$ de solution à 3 p. 100 de triflumuron sur un groupe de 200 mâles, soit en moyenne moins de $1 \mu \mathrm{g} /$ mouche. Le groupe de mâles ainsi traités et le groupe témoin traité à l'eau ont été accouplés successivement à trois groupes de femelles comme précédemment. Les résultats ont montré non seulement des différences très significatives lors des quatre cycles de reproduction du premier accouplement, mais également au deuxième accouplement entre les moyennes des taux d'éclosions $(p<0,001)$. Au premier accouplement, la moyenne des taux d'éclosion lors des quatre premiers cycles chez le lot traité a été de 43,7 \pm 22 p. 100 contre $89,0 \pm 0,5$ p. 100 chez le témoin. Le transfert du triflumuron des mâles traités ( $5 \mu \mathrm{g} /$ mouche) par application topique aux femelles pendant l'accouplement a été peu efficace par rapport à la méthode d'aspersion.
\end{abstract}

1. Institut de l'environnement et de la recherche agricole, 01 BP 910, Bobo-Dioulasso 01, Burkina Faso

Tél. : +226982329; fax : +226970159; e-mail : ziro.bance@coraf.org

2. Laboratoire de biologie et écologie animales, UFR SVT,

université de Ouagadougou, 06 BP 9820, Ouagadougou 06, Burkina Faso Tél. : +226360102; fax : +226307242

3. Project Manager FITCA, OAU/IBAR, PO Box 30786, Nairobi, Kenya E-mail: fitcapm@iconnect.co.ke

4. Centre international de recherche développement

sur l'élevage en zone subhumide,

01 BP 454, Bobo-Dioulasso 01, Burkina Faso

Tél. : +226972053/972287; fax : +226972320

E-mail : cirdes@ird.bf

\section{INTRODUCTION}

Les glossines sont les principaux vecteurs de la trypanosomose animale africaine (TAA). Cette maladie cause à l'économie africaine des pertes considérables $(12,21,23)$. La lutte contre la trypanosomose fait face à différentes contraintes : l'absence de nouvelle molécule de trypanocide et la mauvaise utilisation des médicaments favorisent la création de souches résistantes de trypanosomes $(8,18,19)$; l'importance du rôle des glossines dans la transmission de la maladie rend la lutte antivectorielle incontournable $(8,12)$; enfin, il est nécessaire d'utiliser des méthodes non polluantes pour l'environnement et inoffensives pour l'homme. 
Outre l'utilisation des insecticides sur des supports attractifs, d'autres méthodes alternatives ou complémentaires sont développées (6), visant à empêcher le développement de l'insecte par une action directe ou indirecte sur sa reproduction. Parmi ces méthodes de lutte, figurent les régulateurs de croissance des insectes (IGR), dont la plupart n'ont pas d'influence sur la survie des glossines adultes traitées mais compromettent leurs descendances. Des glossines adultes traitées avec le diflubenzuron, par exemple, ne présentent aucune différence de longévité (16). Des essais conduits au laboratoire sur la descendance des diptères ont montré que les IGR entraînent une chute nette de l'émergence des adultes (9). D'autres essais conduits sur le terrain aboutissent à la même conclusion (4). Le triflumuron fait partie des IGR qui inhibent la synthèse de la chitine et perturbent la métamorphose.

Le triflumuron est sans effet sur l'homme et peu polluant pour l'environnement (3) (Bayer AG Research Center, Monheim, Germany). Son utilisation sur le terrain a déjà montré une certaine efficacité dans la lutte contre les glossines (2). Une dose de $0,5 \mu \mathrm{g}$ dans $1 \mu \mathrm{l}$ d'acétone en application topique sur une femelle de Glossina morsitans morsitans stérilise celle-ci pendant les quatre premiers cycles qui suivent le traitement (14).

La stérilisation a l'avantage d'être très spécifique et permet de viser l'élimination de l'espèce avec efficacité $(13,15)$. Il a été montré qu'il est préférable de stériliser les individus des deux sexes $(10,11,24)$. Il est possible de stériliser les femelles de la glossine par une application directe du triflumuron sur celles-ci (14). Il est également possible d'atteindre la population femelle en traitant les mâles, le produit diffusant lors de l'accouplement. La présente étude, en comparant deux méthodes d'application du triflumuron aux mâles, a cherché à contribuer à éclairer sur la meilleure disposition du triflumuron sur les mâles de la glossine de manière à permettre le transfert du produit aux femelles pendant l'accouplement et ainsi de stériliser les femelles à l'aide de ces mâles, ce qui aboutit à une autostérilisation. Cet objectif a été identique à celui de la technique du lâcher de mâles stériles (5) ou de la lutte autocide.

\section{MATERIEL ET METHODES}

\section{L'insecte}

L'essai a porté sur Glossina palpalis gambiensis Vanderplank, 1949, provenant du laboratoire d'élevage de glossines du Centre international de recherche-développement sur l'élevage en zone subhumide (Cirdes). Cette espèce a été choisie du fait de son importance épidémiologique en Afrique de l'Ouest. Les glossines étaient nourries sur membrane de silicone recouvrant du sang défibriné de bovin ou de porc, à une température de $38 \pm 1^{\circ} \mathrm{C}$. Le repas de sang a duré 10 min à l'abri de la lumière et a été proposé six jours sur sept comme à l'ensemble des lots d'élevage du Cirdes (1).

\section{Le triflumuron}

Le triflumuron, 2-chloro-N-[[[4-(trifluoromethoxy)phenyl]amino] carboxyl]benzamide ou Starycide ${ }^{\circledR}$ sc 480 est fabriqué par la firme Bayer. Le Starycide sc 480 est un inhibiteur de la synthèse de la chitine (3) en empêchant l'insertion de la N-acétyl-glucosamine (Bayer AG Research Center, Monheim, Germany). Le triflumuron agit sur le stade pré-imaginal, particulièrement au moment de la formation de la pupe, et perturbe la métamorphose. Comme la plupart des IGR, son utilisation provoque des malformations des pupes issues des femelles traitées et entraîne la mort de l'embryon (12). Les glossines issues des pupes des femelles traitées meurent à l'éclosion ou quelques jours après (17).

\section{Méthodes d'application}

Deux méthodes ont été utilisées : la méthode topique a consisté à déposer à l'aide d'une micropipette la dose retenue sur la face dorsale du thorax de la glossine mâle. Quatre lots $\left(\mathrm{L}_{0}, \mathrm{~L}_{1}, \mathrm{~L}_{2}, \mathrm{~L}_{3}\right)$ de 150 glossines mâles âgées de six jours dont un lot témoin $\left(\mathrm{L}_{0}\right)$ ont été constitués. Le lot $\mathrm{L}_{0}$ a été traité à l'eau, les groupes $\mathrm{L}_{1}, \mathrm{~L}_{2}$ et $\mathrm{L}_{3}$ respectivement avec $1 \mu \mathrm{g}, 2 \mu \mathrm{g}$ et $5 \mu \mathrm{g}$ de matière active par mouche. Les doses $1 \mu \mathrm{g}, 2 \mu \mathrm{g}$ et $5 \mu \mathrm{g}$ sont des multiples de la dose $0,5 \mu \mathrm{g}$ qui, appliquée directement aux femelles de glossines par la méthode topique, stérilise celles-ci sur les quatre cycles qui suivent les traitements (14). L'âge propice pour l'accouplement de la femelle étant de 3 à 4 jours et celui du mâle de 6 jours $(7,12)$, chaque lot de mâles a été successivement mis en contact avec trois groupes de femelles âgées de trois jours pour accouplement à raison d'un mâle pour une femelle. Le premier contact entre le lot de mâles et le lot de femelles avait lieu immédiatement après le traitement des mâles et durait $48 \mathrm{~h}$. Le deuxième contact avec un nouveau lot de femelles intervenait immédiatement après le premier, soit $48 \mathrm{~h}$ après le traitement au triflumuron des mêmes mâles, et durait également $48 \mathrm{~h}$. Le troisième contact avec un nouveau lot de femelles intervenait à la suite durant $48 \mathrm{~h}$ également.

La seconde méthode a consisté à pulvériser une solution à 3 p. 100 de Starycide sur un groupe de 200 glossines mâles âgées de six jours à raison de $0,8 \mathrm{ml}$ de solution pour 25 mouches contenues dans une cage Roubaud. La pulvérisation a été faite à l'aide d'un pulvérisateur manuel. Le groupe témoin a reçu un brouillard d'eau. Chaque groupe de mâles a été mis successivement en contact avec trois groupes de femelles pour accouplement comme précédemment.

A la fin de chaque appariement de sexe, les femelles ont été séparées et suivies pendant les quatre premiers cycles de reproduction. Les mâles ont été éliminés à l'issue des trois séries d'accouplements.

\section{Paramètres étudiés}

\section{Avortons}

Ont été considérés comme avortons tous les follicules ou toutes les larves expulsées avant terme. L'observation à la loupe binoculaire du contenu des pondoirs a permis de connaître le nombre d'avortons. Les taux d'avortons ont été calculés par rapport au nombre de femelles.

\section{Eclosions}

La perturbation des émergences des adultes est reconnue comme l'effet principal des IGR chez les diptères (4). Les taux d'éclosions ont constitué le paramètre le plus important pour évaluer les effets des deux méthodes sur la reproduction des glossines. La durée de pupaison a été comprise entre 25 et 35 jours à une température moyenne de $25 \pm 1{ }^{\circ} \mathrm{C}$ (12) ; ceci a permis de déterminer le nombre d'émergences des adultes par cycle à partir de l'ensemble des pupes des quatre cycles mises dans des cages d'éclosion. Les taux d'éclosions ou taux d'émergences ont été calculés en rapport avec le nombre de femelles en ponte suivant la formule :

$\mathrm{T}_{\mathrm{ecl}}=\left(\mathrm{E} / \mathrm{F}_{\text {ponte }}\right) \times 100$, où $\mathrm{T}_{\text {ecl }}=$ taux d'éclosions, $\mathrm{E}=$ nombre d'éclosions, $\mathrm{F}_{\text {ponte }}=$ nombre de femelles en ponte.

\section{Malformations des pupes}

Le nombre de pupes mal formées a été obtenu par comptage. A été considérée comme pupe mal formée celle dont la paroi a été très fragile, enflée par endroit, ayant eu une forme plus ou moins ramassée bien différente de celle de la pupe normale. Trois types de malformation ont été identifiés : types I, IIa, IIb. 


\section{Poids des pupes}

La valeur $25 \mathrm{mg}$, poids moyen des pupes de Glossina p. gambiensis dans les élevages du Cirdes, a été choisie comme référence. Toutes les pupes ont été pesées et classées en deux groupes : celles d'un poids inférieur à $25 \mathrm{mg}$ et celles d'un poids supérieur ou égal à $25 \mathrm{mg}$. Les résultats ont été exprimés en pourcentage pour chaque catégorie de pupes par rapport au nombre total de pupes pondues.

\section{Analyse statistique}

Le test du $\chi^{2}$ (20) a été utilisé pour comparer les valeurs des paramètres de reproduction des lots traités entre eux et à celles des témoins.

\section{RESULTATS}

\section{Effectifs}

Le nombre de femelles en ponte suivies a été compris entre 61 et 107 par lot pour le protocole de la méthode topique et entre 114 et 163 par lot pour celui de la méthode de pulvérisation. Pour une même méthode de traitement (topique ou par pulvérisation), les différences entre les effectifs des femelles en ponte ont été dues à la variation des taux de mortalité par lot.

\section{Effets sur les avortons des deux méthodes d'application du triflumuron}

\section{Méthode topique}

$\mathrm{Au}$ premier accouplement, lors du premier cycle, des différences significatives ont été observées entre les doses $(1 \mu \mathrm{g}, 2 \mu \mathrm{g}$ et $5 \mu \mathrm{g})$ et le témoin $(\mathrm{p}<0,50)$ (tableau I). En revanche, il n'y a pas eu de différence entre les trois doses $(1 \mu \mathrm{g}, 2 \mu \mathrm{g}$ et $5 \mu \mathrm{g})$ au risque de 5 p. 100 . Durant les $2^{\mathrm{e}}$ et $4^{\mathrm{e}}$ cycles, seule la dose de $5 \mu \mathrm{g}$ a augmenté significativement les taux d'avortons par rapport au témoin (respectivement $\mathrm{p}<0,05$ et $\mathrm{p}<0,001$ ). Sur l'ensemble des cycles, la dose de $5 \mu \mathrm{g} /$ mouche a augmenté significativement le taux moyen d'avortons $(18,8 \pm 6,1$ p. 100) par rapport au taux moyen d'avortons du témoin $(4,25 \pm 0,96$ p. 100) $(\mathrm{p}<0,01)$, à celui de la dose de $1 \mu \mathrm{g}(7,25 \pm 4,57$ p. 100) $(\mathrm{p}<0,05)$ et à celui de la dose de $2 \mu \mathrm{g}\left(7,5 \pm 4,8\right.$ p. 100) $(\mathrm{p}<0,05)$ (tableau I). Aux $2^{\mathrm{e}}$ et $3^{\mathrm{e}}$ accouplements des mâles, il n'y a pas eu de différence significative entre les taux d'avortons des lots traités et ceux des témoins au seuil de 5 p. 100.

\section{Méthode de pulvérisation}

Au premier accouplement des mâles, il y a eu des différences significatives lors des $1^{\text {er }}$ et $2^{\mathrm{e}}$ cycles, les taux d'avortons du lot traité $(30,2$ p. 100 et 15,1 p. 100) ont été significativement différents de ceux du lot témoin $(8,8$ p. 100 et 4,9 p. 100) (respectivement $\mathrm{p}<0,001$ et $\mathrm{p}<0,01)$. Aux $3^{\mathrm{e}}$ et $4^{\mathrm{e}}$ cycles il n'y a plus eu de différence significative au seuil de 5 p. 100 (tableau II). Aux $2^{\mathrm{e}}$ et $3^{\mathrm{e}}$ accouplements des mâles, il n'y a pas eu de différences significatives entre les taux des avortons des lots traités et ceux des lots témoin au seuil de 5 p. 100.

\section{Effets sur les éclosions des deux méthodes d'application du triflumuron}

\section{Méthode topique}

Au premier accouplement des mâles lors du premier cycle, les doses de $2 \mu \mathrm{g}$ et de $5 \mu \mathrm{g}$ ont réduit significativement les taux d'éclosions $(24,4$ et 24,7 p. 100$)$ par rapport au témoin $(71,8$ p. 100$)$ (p < $<0,05)$ (tableau III). Le taux d'éclosions des pupes issues des femelles

\section{Tableau I}

Evolution des taux d'avortons (\%) chez les femelles du premier accouplement avec les mâles traités à différentes doses de triflumuron par la méthode topique

\begin{tabular}{lcrrr} 
Rang des cycles & Témoin & \multicolumn{3}{c}{ Quantité de triflumuron } \\
\cline { 2 - 5 } & & $\mathbf{1} \boldsymbol{\mu g}$ & $\mathbf{2} \boldsymbol{\mu g}$ & $\mathbf{5} \boldsymbol{\mu g}$ \\
\hline $1^{\mathrm{er}}$ & $4^{\mathrm{b}}$ & $12^{\mathrm{a}}$ & $11^{\mathrm{a}}$ & $16^{\mathrm{a}}$ \\
$2^{\mathrm{e}}$ & $3^{\mathrm{b}}$ & $1^{\mathrm{b}}$ & $2^{\mathrm{b}}$ & $12^{\mathrm{a}}$ \\
$3^{\mathrm{e}}$ & $5^{\mathrm{b}}$ & $8^{\mathrm{b}}$ & $12^{\mathrm{a}}$ & $21^{\mathrm{a}}$ \\
$4^{\mathrm{e}}$ & $5^{\mathrm{b}}$ & $8^{\mathrm{b}}$ & $5^{\mathrm{b}}$ & $26^{\mathrm{a}}$ \\
Moyenne & $4,25 \pm$ & $7,25 \pm$ & $7,5^{ \pm}$ & $18,8 \pm$ \\
& $0,96^{\mathrm{b}}$ & $4,57^{\mathrm{b}}$ & $4,8^{\mathrm{b}}$ & $6,08^{\mathrm{a}}$
\end{tabular}

a, b Sur une même ligne les nombres suivis des mêmes lettres ne sont pas significativement différents au seuil de 5 p. 100 (test du $\chi^{2}$ )

\section{Tableau II}

Evolution des taux d'avortons (\%) issus des femelles du premier accouplement avec les mâles traités par pulvérisation

\begin{tabular}{lcc} 
Rang des cycles & Témoin & Traité \\
\hline $1^{\mathrm{er}}$ & $8,8^{\mathrm{b}}$ & $30,2^{\mathrm{a}}$ \\
$2^{\mathrm{e}}$ & $4,9^{\mathrm{b}}$ & $15,1^{\mathrm{a}}$ \\
$3^{\mathrm{e}}$ & $14,4^{\mathrm{a}}$ & $14,8^{\mathrm{a}}$ \\
$4^{\mathrm{e}}$ & $11,4^{\mathrm{a}}$ & $11,1^{\mathrm{a}}$ \\
Moyenne & $9,9 \pm 4,0^{\mathrm{a}}$ & $17,8 \pm 8,5^{\mathrm{a}}$
\end{tabular}

a, b Sur une même ligne les nombres suivis des mêmes lettres ne sont pas significativement différents au seuil de 5 p. 100 (test du $\chi^{2}$ )

\section{Tableau III}

Evolution des taux d'éclosions (\%) chez les femelles du premier accouplement avec des mâles ayant reçu les différentes doses de triflumuron par la méthode topique

\begin{tabular}{llccc} 
Rang des cycles & Témoin & \multicolumn{3}{c}{ Quantité de triflumuron } \\
\cline { 2 - 5 } & & $\mathbf{1} \boldsymbol{\mu g}$ & $\mathbf{2} \boldsymbol{\mu g}$ & $\mathbf{5} \boldsymbol{\mu g}$ \\
\hline $1^{\mathrm{er}}$ & $71,8^{\mathrm{b}}$ & $60,9^{\mathrm{b}}$ & $24,4^{\mathrm{a}}$ & $24,7^{\mathrm{a}}$ \\
$2^{\mathrm{e}}$ & $79^{\mathrm{a}}$ & $79,8^{\mathrm{a}}$ & $80,6^{\mathrm{a}}$ & $79,6^{\mathrm{a}}$ \\
$3^{\mathrm{e}}$ & $80,7^{\mathrm{a}}$ & $79,5^{\mathrm{a}}$ & $80,6^{\mathrm{a}}$ & $80,6^{\mathrm{a}}$ \\
$4^{\mathrm{e}}$ & $76,7^{\mathrm{a}}$ & $74,8^{\mathrm{a}}$ & $81,3^{\mathrm{a}}$ & $80,3^{\mathrm{a}}$ \\
Moyenne & $77 \pm$ & $73,7 \pm$ & $66,7 \pm$ & $66,3 \pm$ \\
& $4^{\mathrm{a}}$ & $9^{\mathrm{a}}$ & $28^{\mathrm{a}}$ & $28^{\mathrm{a}}$ \\
\end{tabular}

a, b Sur une même ligne les nombres suivis des mêmes lettres ne sont pas significativement différents au seuil de 5 p. 100 (test du $\chi^{2}$ ) 
accouplées avec des mâles ayant reçu $1 \mu \mathrm{g} /$ mouche $(60,9$ p. 100) n'a pas été significativement différent de celui du témoin $(71,8$ p. 100) ( $p>0,05$ ). Aux $2^{\mathrm{e}}, 3^{\mathrm{e}}$ et $4^{\mathrm{e}}$ cycles du $1^{\mathrm{er}}$ accouplement il n'y a pas eu de différence significative entre les taux d'éclosions (tableau III). Aux $2^{\mathrm{e}}$ et $3^{\mathrm{e}}$ accouplements des mâles, il n'y a plus eu de différences significatives au niveau des éclosions chez les lots traités par rapport au témoin au seuil de 5 p. 100.

\section{Méthode de pulvérisation}

Au premier accouplement, lors des quatre cycles de reproduction, il y a eu des différences très significatives entre les taux des éclosions du lot traité et ceux du témoin $(\mathrm{p}<0,001)$. Au deuxième accouplement des mâles, la moyenne $(72,8 \pm 29$ p. 100) des taux d'éclosions des quatre cycles chez le lot traité a été significativement différente de celle $(90,3 \pm 1,7$ p. 100) du lot témoin ( $p<0,001)$ (tableau IV). Cette différence a été essentiellement liée aux taux d'éclosions observés au cours du premier cycle. Au troisième accouplement des mâles, il n'y a eu aucune différence significative entre les taux d'éclosions chez le lot traité et chez le lot témoin au seuil de 5 p. 100.

\section{Effets sur les malformations des pupes des deux méthodes d'application du triflumuron}

\section{Méthode topique}

Aux $1^{\mathrm{er}}, 2^{\mathrm{e}}$ et $3^{\mathrm{e}}$ accouplements, il n'y a pas eu de différences significatives dans les proportions de pupes mal formées chez les lots traités par rapport au témoin (au seuil de 5 p. 100). Les malformations rencontrées ont été de type I (figure 1) et de type II (figure 2). Le type I, avec une surface en forme d'escalier, a été observé au cours des premiers cycles de reproduction du premier accouplement pour les doses $2 \mu \mathrm{g}$ et $5 \mu \mathrm{g}$. Les malformations de type II sont des formes profondes de déformation, parmi lesquelles on peut distinguer deux sous-types, a et $b$, suivant les degrés de malformation. Le type II est donc une forme plus grave de déformation de la paroi de la pupe. La figure 3 montre, à titre comparatif, des pupes apparemment normales.

\section{Méthode de pulvérisation}

Aux $1^{\text {er }}, 2^{\mathrm{e}}$ et $3^{\mathrm{e}}$ accouplements des mâles, il n'y a pas eu de différences significatives entre les proportions des malformations chez le lot traité et chez le lot témoin au risque de 5 p. 100.

\section{Tableau IV}

Evolution des taux d'éclosions (\%) chez les femelles des $1^{\mathrm{er}}$ et $2^{\mathrm{e}}$ accouplements avec les mâles traités par la méthode de pulvérisation

\begin{tabular}{lccccc} 
Rang des cycles & \multicolumn{2}{c}{$\mathbf{1}^{\mathrm{er}}$ accouplement } & & \multicolumn{2}{c}{$\mathbf{2}^{\mathrm{e}}$ accouplement } \\
\cline { 2 - 5 } & Témoin & Traité & & Témoin & Traité \\
\hline $1^{\mathrm{er}}$ & $88^{\mathrm{b}}$ & $13^{\mathrm{a}}$ & $88^{\mathrm{b}}$ & $29^{\mathrm{a}}$ \\
$2^{\mathrm{e}}$ & $90^{\mathrm{b}}$ & $43^{\mathrm{a}}$ & $92^{\mathrm{a}}$ & $87^{\mathrm{a}}$ \\
$3^{\mathrm{e}}$ & $89^{\mathrm{b}}$ & $58^{\mathrm{a}}$ & $91^{\mathrm{a}}$ & $88^{\mathrm{a}}$ \\
$4^{\mathrm{e}}$ & $89^{\mathrm{b}}$ & $60^{\mathrm{a}}$ & $90^{\mathrm{a}}$ & $87^{\mathrm{a}}$ \\
Moyenne & $89 \pm$ & $43,5 \pm$ & $90,3 \pm$ & $72,8 \pm$ \\
& $0,82^{\mathrm{b}}$ & $21,7^{\mathrm{a}}$ & $1,71^{\mathrm{b}}$ & $29,2^{\mathrm{a}}$ \\
\end{tabular}

a, b Sur une même ligne les nombres suivis des mêmes lettres ne sont pas significativement différents au seuil de 5 p. 100 (test du $\chi^{2}$ )

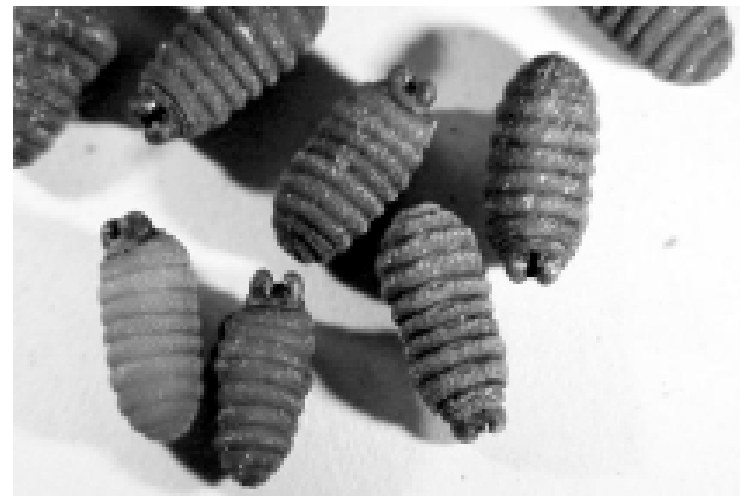

Figure 1 : type I de malformations (parois en escalier) des pupes issues des femelles accouplées avec des mâles traités par pulvérisation d'une solution de $3 \mathrm{p} .100$ de triflumuron ou par application topique (2 $\mu \mathrm{g}, 5 \mu \mathrm{g} /$ mouche mâle).

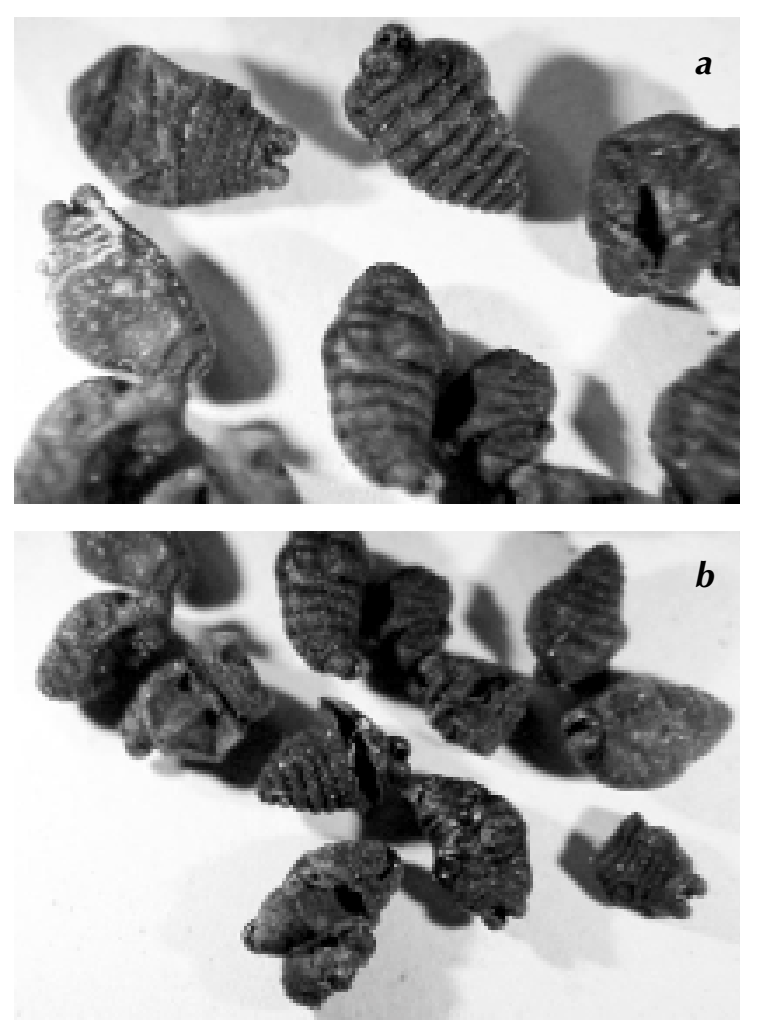

Figure 2 : type II de malformations (a et b) des pupes issues des femelles accouplées avec des mâles traités par pulvérisation d'une solution de 3 p. 100 de triflumuron ou par application topique (2 $\mu \mathrm{g}, 5 \mu \mathrm{g} /$ mouche mâle).

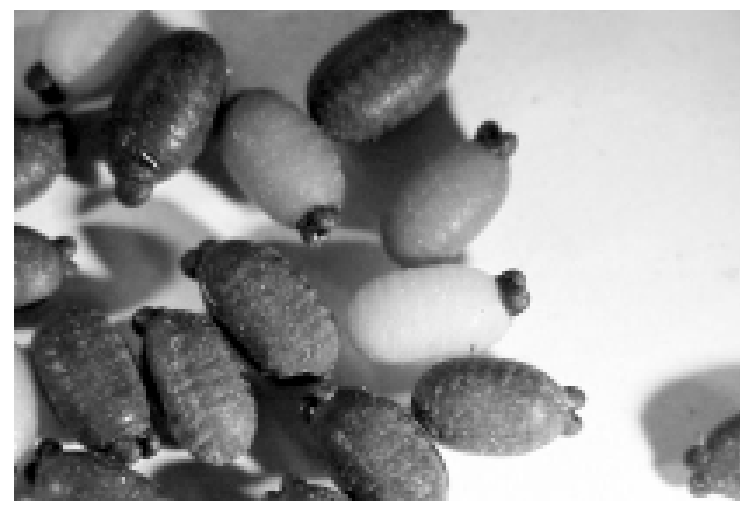

Figure 3 : pupes normales ou apparemment normales. 


\section{Effets sur le poids des pupes des deux méthodes d'application du triflumuron}

\section{Méthode topique}

Au $1^{\mathrm{er}}$ accouplement lors des $1^{\mathrm{e} r}, 2^{\mathrm{e}}$ et $3^{\mathrm{e}}$ cycles de reproduction, les doses de $2 \mu \mathrm{g}$ et de $5 \mu \mathrm{g}$ ont réduit significativement le pourcentage de pupes dont le poids a été supérieur ou égal à $25 \mathrm{mg}$ par rapport au témoin et à la dose de $1 \mu \mathrm{g}$. Au $4^{\mathrm{e}}$ cycle, seule la dose de $5 \mu \mathrm{g}$ a réduit encore significativement ce pourcentage. En revanche, au cours du $4^{\mathrm{e}}$ cycle, il n'y a pas eu de différence significative entre les doses de $1 \mu \mathrm{g}$, de $2 \mu \mathrm{g}$ et le témoin au seuil de $5 \mathrm{p}$. 100. Au niveau des moyennes des quatre cycles de reproduction, les doses de $2 \mu \mathrm{g}$ et de $5 \mu \mathrm{g}$ de triflumuron par mouche mâle ont réduit significativement le pourcentage de pupes dont le poids a été supérieur ou égal à $25 \mathrm{mg}$ par rapport au témoin $(2 \mu \mathrm{g} v s$ témoin, $\mathrm{p}<0,01)$, $(5 \mu \mathrm{g} v s$ témoin, $\mathrm{p}<0,001)$, sans différence significative entre elles. Pour la dose de $1 \mu \mathrm{g}$, la moyenne $(76,7 \pm 8 \mathrm{p} .100)$ des pourcentages des pupes dont le poids a été supérieur ou égal à $25 \mathrm{mg}$ n'a pas été significativement différente de celle $(73,5 \pm 7$ p. 100$)$ du témoin $\left(\chi^{2}=0,33, p>0,05\right)$ (tableau V). Aux $2^{\mathrm{e}}$ et $3^{\mathrm{e}}$ accouplements des mâles, il n'y a eu aucune différence significative entre les pourcentages de pupes dont le poids a été supérieur ou égal à $25 \mathrm{mg}$ au seuil de 5 p. 100 .

\section{Méthode de pulvérisation}

$\mathrm{Au}$ premier accouplement, le triflumuron a entrainé une baisse significative des pourcentages de pupes dont le poids a été supérieur ou égal à $25 \mathrm{mg}$ entre le lot traité et le témoin au $1^{\mathrm{er}}$ cycle $(p<0,001)$ et au $2^{\mathrm{e}}$ cycle $(\mathrm{p}<0,05)$. Il n'y a plus eu de différence aux $3^{\mathrm{e}}$ et $4^{\mathrm{e}}$ cycles (tableau VI). Aux $2^{\mathrm{e}}$ et $3^{\mathrm{e}}$ accouplements des mâles il n'y a pas eu de différence significative entre les lots traités et les lots témoins au risque de 5 p. 100.

\section{DISCUSSION}

L'effet du triflumuron sur la proportion des avortons a été moins important chez les lots traités par pulvérisation que par application topique. Selon la littérature, des doses importantes de triflumuron provoquent de forts taux d'avortement (14). C'est lors du premier cycle de reproduction que l'effet du triflumuron a été le plus visible, entraînant des différences significatives entre le taux d'avortons du lot traité $(30,2$ p. 100) et celui du lot témoin $(8,8$ p. 100). Les différences n'ont pas été significatives pour les cycles suivants, mais une augmentation inexpliquée des taux d'avortons est apparue au cours des $3^{\mathrm{e}}$ et $4^{\mathrm{e}}$ cycles de reproduction dans le lot témoin de la méthode de pulvérisation. La baisse des taux d'avortons aux $2^{\mathrm{e}}, 3^{\mathrm{e}}$ et $4^{\mathrm{e}}$ cycles du lot traité pouvait être attribuée à la diminution de l'effet du triflumuron avec le rang des cycles. Par ailleurs, entre la méthode de traitement par aspersion et celle en application topique de $5 \mu \mathrm{g}$ de triflumuron, l'augmentation globale des taux d'avortons sur l'ensemble des quatre cycles a semblé identique. La pulvérisation a eu le même effet que l'application topique, mais à une dose cinq fois moins importante. Cette dernière est apparue moins efficace que la méthode d'aspersion.

Les éclosions ont constitué le paramètre le plus important pour comparer les effets du triflumuron à travers les deux méthodes d'application. Avec la méthode topique, les doses les plus élevées ( $2 \mu \mathrm{g} / \mu \mathrm{l} / \mathrm{mouche}$ et $5 \mu \mathrm{g} / \mu \mathrm{l} / \mathrm{mouche}$ ) appliquées aux mâles ont entraîné une chute significative des taux d'éclosions des pupes limitée au premier cycle du premier accouplement. Avec la méthode de pulvérisation, le triflumuron a entraîné une baisse nette des éclosions après les premier et second accouplements des mâles. La quantité de produit transmise par le mâle traité par la méthode topique à la femelle pendant l'accouplement aurait été très faible. Les doses $2 \mu \mathrm{g}$ et $5 \mu \mathrm{g}$ par mouche représentaient quatre

\section{Tableau V}

Pourcentage de production de pupes dont le poids est supérieur ou égal à $25 \mathrm{mg}$ issues des femelles après le premier accouplement avec

les mâles traités à différentes doses

de triflumuron par la méthode topique

\begin{tabular}{lllll} 
Rang des cycles & Témoin & \multicolumn{3}{c}{ Quantité de triflumuron } \\
\cline { 2 - 5 } & & $\mathbf{1} \boldsymbol{\mu g}$ & $\mathbf{2} \boldsymbol{\mu g}$ & $\mathbf{5} \boldsymbol{\mu g}$ \\
\hline $1^{\mathrm{er}}$ & $78,6^{\mathrm{b}}$ & $67,6^{\mathrm{b}}$ & $47,5^{\mathrm{a}}$ & $40,5^{\mathrm{a}}$ \\
$2^{\mathrm{e}}$ & $77,3^{\mathrm{b}}$ & $85,9^{\mathrm{b}}$ & $66,7^{\mathrm{a}}$ & $56,3^{\mathrm{a}}$ \\
$3^{\mathrm{e}}$ & $74,3^{\mathrm{b}}$ & $78,8^{\mathrm{b}}$ & $53,1^{\mathrm{a}}$ & $53,7^{\mathrm{a}}$ \\
$4^{\mathrm{e}}$ & $63,6^{\mathrm{b}}$ & $73^{\mathrm{b}}$ & $66,7^{\mathrm{b}}$ & $45,3^{\mathrm{a}}$ \\
Moyenne & $73,5^{ \pm}$ & $76,3^{ \pm}$ & $58,8 \pm$ & $49 \pm$ \\
& $6,8^{\mathrm{b}}$ & $7,8^{\mathrm{b}}$ & $9,7^{\mathrm{a}}$ & $7,3^{\mathrm{a}}$ \\
\end{tabular}

a, b Sur une même ligne les nombres suivis des mêmes lettres ne sont pas significativement différents au seuil de 5 p. 100 (test du $\chi^{2}$ )

\section{Tableau VI}

Pourcentage de pupes dont le poids est supérieur ou égal à $25 \mathrm{mg}$ issues des femelles accouplées avec les mâles traités par pulvérisation au premier accouplement

\begin{tabular}{lll} 
Rang des cycles & Témoin & Traité \\
\hline $1^{\mathrm{er}}$ & $50^{\mathrm{b}}$ & $23,4^{\mathrm{a}}$ \\
$2^{\mathrm{e}}$ & $56,2^{\mathrm{b}}$ & $45^{\mathrm{a}}$ \\
$3^{\mathrm{e}}$ & $69^{\mathrm{a}}$ & $67^{\mathrm{a}}$ \\
$4^{\mathrm{e}}$ & $58^{\mathrm{a}}$ & $57^{\mathrm{a}}$ \\
Moyenne & $58,3 \pm 9^{\mathrm{a}}$ & $50,7 \pm 23^{\mathrm{a}}$ \\
\hline
\end{tabular}

a, b Sur une même ligne les nombres suivis des mêmes lettres ne sont pas significativement différents au seuil de 5 p. 100 (test du $\chi^{2}$ )

et dix fois la dose qui, appliquée directement à la femelle, stérilise celle-ci pendant les quatre cycles de reproduction qui suivent le traitement (14). La transmission du produit à la femelle pendant l'accouplement dépend de la position du produit sur le corps du mâle traité. Lors de la présente étude, le lieu d'application du produit (face dorsale du thorax) n'aurait pas permis un contact direct du produit avec la femelle pendant l'accouplement, ce qui pouvait expliquer la diffusion très faible.

Avec la méthode d'aspersion, la dose transmise du mâle à la femelle a baissé avec le rang d'accouplement, entraînant de moins en moins de perturbations. Le mâle traité par aspersion a ainsi été capable de transmettre une dose active du produit à la femelle au cours des 96 heures qui ont suivi le traitement. Par ailleurs, la réduction des taux d'éclosions par le triflumuron a baissé avec le rang du cycle des glossines femelles contaminées. Ces informations sont très importantes pour préciser la dose à appliquer.

Concernant la forme des pupes, les types I et II de malformation ont été observés pour les deux méthodes de traitement, avec les doses topiques les plus élevées ( $2 \mu \mathrm{g} /$ mouche et $5 \mu \mathrm{g} /$ mouche) et avec l'aspersion. Les malformations rencontrées ont été conformes à celles déjà signalées par le Tsetse Research Laboratory (22). Selon ce rapport, ces malformations de pupes de glossines sont 
propres aux IGR. Ces types de déformation pourraient caractériser le degré de contamination des femelles par le triflumuron.

La variation du poids des pupes a été très liée à la malformation des pupes. Les pupes mal formées (types IIa et IIb) pesaient généralement moins lourd que les pupes ordinaires normales. La membrane protectrice des pupes mal formées était très fragile et se déchirait au moindre choc. Elles devaient être récoltées à un intervalle de 48 heures afin de pouvoir être pesées. Plus la malformation a été importante, plus la pupe a été légère en raison de la perte d'une partie de son contenu liquide par déshydratation.

\section{- CONCLUSION}

Les glossines mâles traitées par la méthode topique sur le thorax, même à la dose de $5 \mu \mathrm{g}$ de triflumuron par microlitre et par mouche, ont transféré très peu de produit aux femelles au cours de l'accouplement : les émergences d'adultes n'ont été significativement perturbées qu'au $1^{\mathrm{er}}$ cycle du $1^{\mathrm{er}}$ accouplement. Cette dose a représenté pourtant 10 fois celle qui, appliquée directement à la femelle, a stérilisé celle-ci sur les quatre cycles de reproduction qui ont suivi le traitement.

\section{BIBLIOGRAPHIE}

1. BAUER B., POLITZAR H., 1982. Laboratory maintenance of Glossina palpalis gambiensis in West Africa. Preliminary results of rearing on membranes. Vienna, Austria, IAEA, p. 225-263.

2. BAUER B., KABORE I., LEFRANÇOIS T., SOLANO P., 1997. Impact of the chitin synthesis inhibitor triflumuron on two tsetse species in the subhumid zone of Burkina Faso, West Africa. In: 24th Meeting of OAU/ISCTRC, Maputo, Mosambique, p. 348.

3. BAYER, 1993. Technical information (BAY SIR 8514); chitin synthesis inhibitor particularly for the control of biting pests. Monheim, Germany, Bayer.

4. CHEVASSE D.C., LINES J.D., ICHIMORI K., MAJALA A.R., MINJAS J.N., MARIJANI J., 1995. Mosquito control in Dar el Salaam. II. Impact of expanded polystyrene beads and pyriproxyfen treatment of breeding sites on Culex qunquefasciatus densities. Med. vet. Entomol., 9: 147-154.

5. CUISANCE D., POLITZAR H., MEROT P., TAMBOURA I., 1984. Les lâchers de mâles irradiés dans la campagne de lutte intégrée contre les glossines dans la zone pastorale de Sidéradougou (Burkina Faso). Revue Elev. Méd. vét. Pays trop., 37 : 449-467.

6. CUISANCE D., BARRE N., DE DEKEN R., 1994. Ectoparasites des animaux : méthodes de lutte écologique, biologique, génétique et mécanique. Revue sci. tech. Off. int. Epizoot., 13 : 1305-1356.

7. DAVIES H., 1967. Tsetse flies in Northern Nigeria. Ibadan, Nigeria, Ibadan University Press, $148 \mathrm{p}$.

8. GEERTS S., HOLMES P.H., 1997. Gestion médicamenteuse et résistance parasitaire. In : 24e réunion OUA/CSRLT, Maputo, Mozambique. Nairobi, Kenya, OAU/ISTRC, p. 371-385. (Publication $\mathrm{n}^{\circ}$ 119)

9. HALOS L., 2001. Lutte contre les glossines au Burkina Faso influence $d^{\prime} u n$ régulateur de croissance des insectes, le triflumuron, sur la compétence vectorielle de deux espèces de glossines riveraines et application à un protocole de lutte ciblée. DESS Productions animales en régions chaudes, université Montpellier II, France, 52 p. (Rapport de stage)

10. HOWARD J.J., WALL R., 1995. The effects of triflumuron, a chitin synthesis inhibitor, on the house fly, Musca domestica (Diptera: Muscidae). Bull. entomol. Res., 85: 71-77.

11. HOWARD J., WALL R., 1996. Autosterilisation of the house fly Musca domestica (Diptera: Muscidae) in poultry houses in North-East India. Bull. Entomol. Res., 86: 363-367.
En revanche, les mâles traités en aspersion à une dose légèrement inférieure à $1 \mu \mathrm{g}$ par mouche ont transmis aux femelles des doses de triflumuron qui ont réduit significativement l'émergence de la descendance des mouches pendant les quatre cycles aux $1^{\mathrm{er}}$ et $2^{\mathrm{e}}$ accouplements. Cette étude montre que la localisation du produit sur le corps des mâles joue un rôle important dans le transfert du produit des mâles traités aux femelles pendant l'accouplement. La méthode de traitement par aspersion laisse entrevoir la possibilité de stériliser les femelles par les mâles traités et de réaliser ainsi une autostérilisation au sein de la population glossinienne à l'aide du triflumuron. Ces résultats montrent qu'il est maintenant important de réaliser des applications topiques sur les parties inférieures (tarse, abdomen) de l'insecte, parties qui sont en contact avec les tissus imprégnés.

\section{Remerciements}

Ce travail a été réalisé grâce au concours matériel et financier du Cirdes. Les auteurs tiennent à exprimer à la direction de ce centre toute leur gratitude. Ils remercient également $\mathrm{Z}$. Bengaly, W. Yoni, L. Sanogo du Cirdes et Dr D. Dakouo, entomologiste à l'Inera Bobo-Dioulasso, pour leur assistance.
12. ITARD J., 1986. Les glossines ou mouches tsé-tsé. Maisons-Alfort, France, lemvt, 155 p. (Etudes et synthèses $n^{\circ} 15$ )

13. LANGLEY P.A., WEIDHAAS D., 1986. Trapping as a means of controlling tsetse, Glossina spp. (Diptera: Glossinidae): the relative merits of killing and of sterilisation. Bull. entomol. Res., 76: 89-95.

14. LANGLEY P.A., 1995. Evaluation of the chitin synthesis inhibitor triflumuron for controlling the tsetse Glossina m. morsitans (Diptera: Glossinidae). Bull. entomol. Res., 85: 495-500.

15. LANGLEY P.A., 1997. Autosterilization as a means of tsetse control: a role for insect growth regulators (IGRs). In: 24th meeting of OAU/ISCTRC, Maputo, Mozambique. Nairobi, Kenya, OAU/ISTRC, p. 343-347.

16. MOURET J., 1983. Effets démographiques du diflubenzuron sur la mouche tsé-tsé. Cah. Orstom, Sér. Ent. Méd. Parasitol., 21 : 19-27.

17. OUEDRAOGO S., 1999. Contribution à l'étude de l'efficacité et de la rémanence du triflumuron en pulvérisation sur tissu pour I'amélioration des techniques de lutte non polluantes contre les glossines ou mouche tsé-tsé (essai sur Glossina palpalis gambiensis). Mémoire fin d'études, IDR/UP, Bobo Dioulasso, Burkina Faso, 65 p.

18. PEREGRINE A.S., 1994. Chemotherapy and delivery systems: haemoparasites. Vet. Parasitol., 54: 223-248.

19. RAYNAUD J.P., SONES K.R., FRIEDHEIM E.A.H., 1989. A review of Cymelarsan ${ }^{\circledR}$, a new trypanocide proposed for the treatment of $T$. evansi infections. In: 20th Meeting OAU/ISCTRC, Mombasa, Kenya, p. 334-338.

20. SCHWARTZ D., 1980. Méthodes statistiques à I'usage des médecins et des biologistes, $3^{e}$ édn. Paris, France, Flammarion médecine sciences, 318 p. (Coll. Statistique en biologie et en médecine)

21. SWALLOW B.M., 1997. Impacts of trypanosomosis on African agriculture. In: 24th Meeting OAU/ISCTRC, Maputo, Mozambique, p. 515-536. (Publication $\mathrm{n}^{\circ} 119$ )

22. The tsetse research laboratory, 1979. Bristol, UK, Langford, $19 \mathrm{p}$

23. TOURE S.M., MORTELMANS J., 1991. Impact de la trypanosomose animale africaine. Bull. Séances Acad. R. Sci. Outre-Mer, 36 : 239-257.

24. WALL R., HOWARD J., 1994. Autosterilisation for the control of the house fly, Musca domestica. J. theor. Biol., 171: 431-437.

Accepté le 12.04.2004 


\section{Summary}

Bancé Z.A., Ouédraogo P.A., Bauer B., Kaboré I., Sidibé I. Two Triflumuron Application Methods on Glossina palpalis gambiensis in the Laboratory: Impact on Female Fertility

The objective of this study was to compare the efficacy of two triflumuron-application methods on male tsetse flies to achieve transfer of the product to the females during subsequent mating. For the topical application, which consisted in applying the solution to the upper part of the thorax, four groups of six-day-old male tsetse flies received $1 \mu \mathrm{g}, 2 \mu \mathrm{g}, 5 \mu \mathrm{g}$ triflumuron in $1 \mu \mathrm{l}$ water, and water only for the control group, respectively. Each male group was mated consecutively with three groups of 150 three-day-old females. The effects assessed were the abortion rate, eclosion rate, and shape and weight of pupa. The analysis of the results of the first four reproductive cycles of the females showed significant differences between the eclosion rates in females mated with males treated at the doses of $2 \mu \mathrm{g}$ and $5 \mu \mathrm{g}$, and eclosion rates in females mated with the control group only during the $1^{\text {st }}$ cycle of the $1^{\text {st }}$ mating $(p<0.05)$. The second method consisted in spraying 200 males with an $0.8 \mathrm{ml}$ solution containing $3 \%$ triflumuron, i.e. less than $1 \mu \mathrm{g}$ triflumuron per fly. Treated males and the control group were then exposed to three groups of females as described above. The results showed not only highly significant differences during the four reproductive cycles of the $1^{\text {st }}$ mating, but also during the $2^{\text {nd }}$ mating in the means of eclosion rates $(p<0.001)$. During the $1^{\text {st }}$ mating the mean of eclosion rates of the first four reproductive cycles was $43.7 \pm 22 \%$ in the treated group vs $89.0 \pm 0.5 \%$ in the control. The transfer of triflumuron ( $5 \mu \mathrm{g} / \mathrm{fly}$ ) to females by males treated with the topic method was little effective compared to that obtained with the spraying method.

Key words: Glossina palpalis gambiensis - Triflumuron Insect growth regulator - Topical application - Spraying Burkina Faso.

\section{Resumen}

Bancé Z.A., Ouédraogo P.A., Bauer B., Kaboré I., Sidibé I. Estudio sobre dos métodos de aplicación del triflumuron sobre los machos de Glossina palpalis gambiensis en laboratorio: impacto sobre la reproducción de las hembras

El objetivo del presento estudio es el de comparar la eficiencia de dos métodos de aplicación del triflumuron sobre las glosinas macho, para el transporte del producto hacia las hembras durante la copulación. El método tópico, con depósito de la solución sobre la parte superior del tórax, fue utilizado en cuatro grupos de 150 glosinas machos de seis días de edad, las cuales recibieron $1 \mu \mathrm{g}, 2 \mu \mathrm{g}$ y $5 \mu \mathrm{g}$ de triflumuron contenidos en $1 \mu \mathrm{l}$ de agua, el testigo recibió agua. Cada grupo de machos fue seguidamente apareado en forma sucesiva con tres grupos de 150 hembras de tres días de edad durante 48 horas. Los efectos medidos fueron la tasa de aborto y de eclosión de las pupas, la forma y el peso de las pupas. El análisis de los resultados sobre los cuatro primeros ciclos reproductivos de las hembras demostró las diferencias significativas entre las tasas de eclosión en las hembras acopladas con los machos tratados con dosis de $2 \mu \mathrm{g}$ y $5 \mu \mathrm{g}$ y las tasas de eclosión en las hembras del lote testigo, únicamente durante el primer ciclo de apareamiento $(p<0,05)$. El segundo método consistió en un aerosol de $0,8 \mathrm{ml}$ de solución a 3\% de triflumuron sobre un grupo de 200 machos, o sea un promedio de menos de $1 \mu \mathrm{g} /$ mosca. El grupo de machos tratados de esta manera y el grupo testigo tratado con agua se acoplaron sucesivamente con tres grupos de hembras, como anteriormente. Los resultados mostraron no solamente las diferencias significativas durante los cuatro ciclos de reproducción durante la primera copulación, sino también durante la segunda copulación entre los promedios de las tasas de eclosión $(p<0,001)$. Durante la primera copulación, el promedio de la tasa de eclosión durante los primeros cuatro ciclos en el lote tratado fue de $43,7 \pm 22 \%$, contra 89,0 $\pm 0,5 \%$ en los testigos. El transporte de triflumuron de los machos tratados $(5 \mu \mathrm{g} /$ mosca) mediante la aplicación tópica a las hembras durante la copulación fue poco eficiente con respecto al método de aerosol.

Palabras clave: Glossina palpalis gambiensis - Triflumurón Inhibidor desarollo insectos - Aplicatión local - Pulverización Burkina Faso. 